\title{
Science, medical practice and ethics in the next millennium
}

$\mathrm{W}$ hy do some patients looks for a doctor and not others? The following explanation would appear to be a reasonable answer to the question. Most patients visit the doctor because they are concerned about some aspect of their health: bodily function, mental integrity, appearance, sleep etc. And they believe that the doctor will certainly reduce the risks. Other do not look for a doctor because they think they do not need one, although often they are afraid that they might hear bad news about their health or be submitted to threatening diagnostic tests or strong medications whose consequences are not easy to predict. This would explain the large number of patients who prefer medical practices which do not have any scientific basis but apparently (and only apparently) are not harmful.

For both types of patient or, in other words, for all patients, and also for the physician's sake, every medical approach must cause more good than harm. This is the basis for all medical practice and ethical relationships. So, every time a decision is made, the practice of medicine requires the balancing of medical approaches against patients' problems in order to prevent errors.

\footnotetext{
* $M D, P h D, M C E$

Chairman, Department of Internal Medicine, Escola

Paulista de Medicina; Editor, São Paulo Medical Journal.
}

For this balance to have some precision, it needs to be solidly supported by good clinical evaluation and medical knowledge of etiology, etiopathogenesis, physiopathology, diagnostic methods and the effectiveness and efficiency of therapeutics.

As we know, medicine is not a exact science, and biological data and statistics only offer us information in terms of probabilities. Thus, the physician's role is to reduce the chances of the patient having an adverse outcome. Medicine based on the best existing evidence gives the doctor data which does not guarantee results but decreases the chances of mistakes.

Patients need to be aware that when the doctor explains the above balancing process in lay terms, adapted to the individual patient's conditions and psychological and cultural attributes, allowing him to participate in the decision, the total risk will decrease and the residual risk will be shared by doctor and patient. If something bad happens, it will already have been predicted.

Throughout this ethical relationship, the use of good evidence reduces medical errors and may retain confidence amongst those patients who come to the doctor with the aim of reducing risks, and stimulate those other patients who are afraid of visits to the doctor to join in with the more confident ones.

That is what developed countries are working towards for medical practice in the next millennium. 